sulcato-immersis, tenuissimis, infra vix prominulis notata. Spatha ante folia exserta, pedunculo brevi insidens, in tubum pollicarem l. paullo longiorem, diametro subsemi polticarem l. vix pollicarem, laxe-obvallantem convoluta, ima basi tantum connata, in laminam patenter-recurvam denuo expansa, oblongo-ovatam $3-4$ pollices longam, sesqui pollicem l. pollices 2 latam, apice ratundato cuspidulo brevi subulato terminatam, antice concavam fusco-atropurpuream, ad latera revoluto - recurvam, postice virentem, lineolis abruptis longulis purpureo-marmoratam, parte convoluta (tubo) triplo et ultra longiorem. Spadix spatha paullo brevior, erectus, infina basi ad 3-4 linearem altitudinem ovariis, ad pollicarem circiter organis rudimentariis, ad bipollicarem altitudinem vero staminibus tectus reliqua parte appendiculari nudus; appendice primum magis cylindracea, tandem infra l. eirca medium incrassata, basin apicemque obtusum versus leviter attenuata. Ovaria plurima, densa, in stylum brevem (ovario duplo breviorem) cylindroideum attenuata, stigmate rotundato terminata. Flores rudimentarii subulati, elongati, (foemineis duplo longiores), inferiores ovariis contigu, magis approximati, superiores distantiores ad antheras usque dispositi. Flores masculi: Antherae bitoculares latiusculae, breves, sessiles, dense-approximatae, apice biporosae.

(Folia ex vivo spadix cum spatha ex sicco.)

H. S c h o t t.

\title{
Ueber die in Griechenland vorkommenden kryp- togamischen Pflanzen und deren Bedeutung bei den alten Griechen.
}

\author{
Von Professor X. Landerer in Athen.
}

Verschiedene Species Equisetum finden sich z.B. E. sylvaticum, $\boldsymbol{E}$. arvense, $\boldsymbol{E}$. fluviatile theils an schattigen Wasserleitungen in der Nähe von Athen und auch in Arkadien, in Elis und seltener auf den Inseln des griechischen Archipels. Das Equisetum wurde bei den

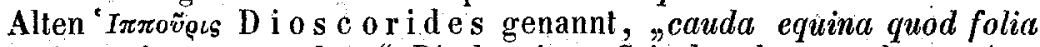
setis equinis respondent." Die heutigen Griechen kennen dessen Anwendung zum Poliren nicht, daher eine Menge dieser Pflanzen aus dem Auslande eingeführt wird.

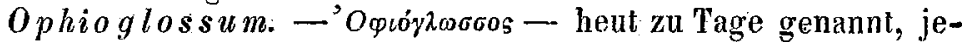
doch höchst selten und ohne Anwendung bei den heutigen Griechen.

Polypodium vulgare. Moגvródıov des Dioscorides. Polypodi heut zn Tage und auch Dendrophtiri. Findet sich in Arkadien häufig an schattigen Felsen und alten Eichen, und wegen der Aehnlichkeit der Blätter und dem Standorte in der Nähe von

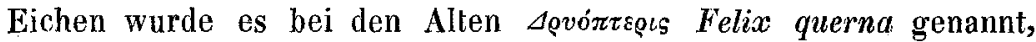
"quae a foliorum figura et loco natali nomen obtinuit," D i o s c o$\mathbf{r}$ ides. Die Alten unterschieden auch das Polypodium Lonchitis von dem Worte Aóyxn Lanze, da dessen Blatt dem dreieckigen, spitzi- 
gen Eisen eines Wurfspiesses ähnlich sieht - „quod folia instar lanceae in acutum desinunt."

Aspidium Filix Mas. Mréprs bèi Dioscorides, - eben-

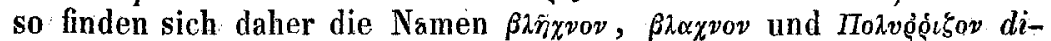
citur cui folia sine caule, sine flore, sine semine. - Irégos ab alis avium dicta.

Das Aspidium filix femina hiess bei den Alten $\Theta \eta \lambda \dot{v} \pi \tau \varepsilon \rho\llcorner$ et Nvequice und Plinins sagt: "Alterum genus thelypterin Graeci vocant, alii nymphaeanpterin." Die heutigen Griechen heissen diese in vielen Theilen Griechenlands sich findende Pflanze Pteri, und schreiben derselben verschiedene Heilkräfte, mehr jedoch auflösende als anthelmintische Wirkungen zu.

Ceterach officinarum. Diese Pflanze hiess bei den $\mathrm{Al}$ -

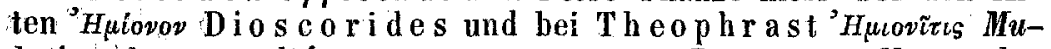

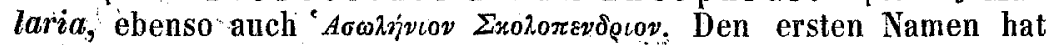
es von dem Maulesel, weil es, von den Wëibern genossen, die Un-

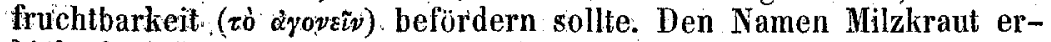
hielt dasselbe wegen des Gebrauches bei Milzleiden, und den Namen Skolopendrion wegen der Aehnlichkeit der Blätter mit der Gestalt

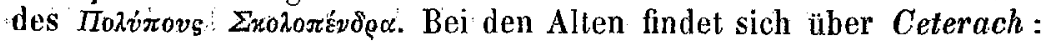
neque caulem, neque isemen, neque florem profert, was jedoch ihrer Unkenntniss in Betreff der Kryptogamen zuzuschreiben ist.

Scolopendrium führt Dioscorides unter dem Namen

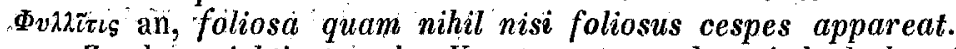

$\mathrm{Zu}$ den wichtigsten der Kryptogamen gehört jedoch das Adiantum Capillus Veneris. Diese Pflanze hatte den folgenden Namen

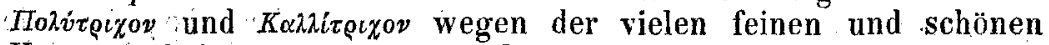
Haare, und A puleg u sagt: "Quod capillos multos ac densos faciat, corumque defluvia expleat, eos tingat pulchrioresque reddat." Das Adiantum findet sich auch mit dem Namen Cincinolis, terrae capillus, supercilium terrae, crinita, jedoch deren Hauptname Adi-

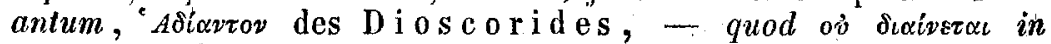
aquis non madescit. Nic a n der sagt: "Quod imbris e coelo descendentis stillicidia foliis ejus non insideant."

Diese Pflanze, Pollytrichi von den heutigen Griechen genannt, ist ein sehr beliebter Thee und vorzüglich bei Frauenkrankheiten im ganzen Oriente sehr gebraucht.

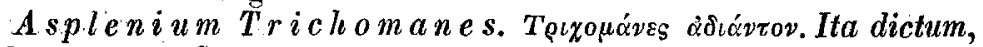
quod rara cute fluentem capillum expleat et subnasci faciat. Diese Kryptogame findet sich sehr selten und soll von den Landleuten als fiebertreibendes Mittel angewendet werden.

Die Moose und Algen wurden bei den Alten B@viov und $\Sigma \pi \lambda \alpha \gamma \chi v o v$ genannt, und P I in i u sagt auch: „Atticis Bryon et Spagnon appellatur."

Unter diesen das bedeutendste war der $\Phi \tilde{v}$ xos des Dios c or i-

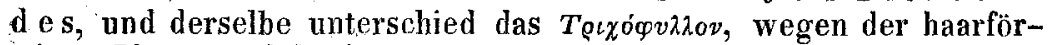

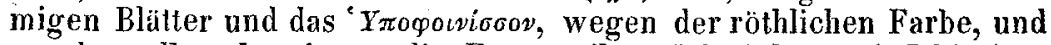
aus demselben bereiteten die Frauen ihre Schminke und Plini us sagt: „Fucus, quo mulieres utuntur ad pingendas malas vitia corporis fuco occullunt." 
Ausserdem kannten die Alten auch das Meergras Zostera marina und Vi r gil sagt: "Alga herba marina, quam dicente Festo, mare ad litus projicit dicta ab algorea quae, vel quod sua crassitie pedes alligat." Die Alten gebrauchten das Meergras zum Ausstopfen von

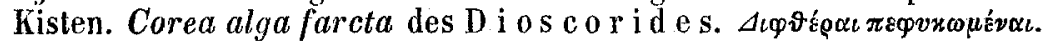

Aus der Classe der Lichenen $A \varepsilon \iota x^{\prime} y$ genannt, und nach Ga le so benannt, weil es an Bäumen, wo ein Ausschlag sich befindet, und die Flechten auf der menschlichen Haut heilen soll. Nach dem Vorkommen nannten die Alten diese Lichenen nach dem Baume, auf

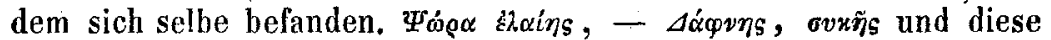
scabies Oleae - Ficus - Lauri wurde als Arzeneimittel gebraucht.

Aus der Classe der Conferven kannten die Alten nur C. lit-

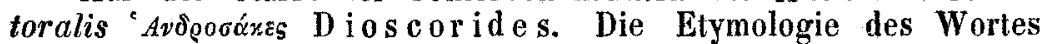
Conferva soll nach Plinius von a conferruminando, das Eisen zusammenlüthen" herkommen, und Androsaces dicitur, quod hydropicorum aquas ducit, vel hydrosacer qui novis in aquis nascitur.

Die Schwämme hiessen bei den Alten Mvंx $\eta$ von $\mu$ ṽros, weil er aus Schleim und Feuchtigkeit besteht, und man unterschied drei Ar-

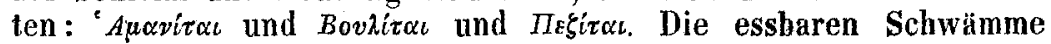
waren bei den Griechen und Römern sehr beliebt, die heutigen Griechen würden keinen Schwamm essen, und uns Fremde verlachen, wenn wir solche essen. Nero boletos cibum deorum vocavit (ad

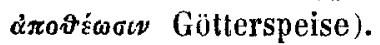

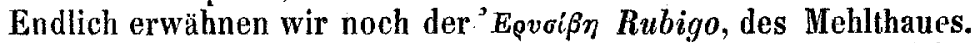
Die Alten kannten den schädlichen Einfluss dieser Krankheit auf das Getreide, und um dieselbe abzuwenden, rief man bei den Rhodiern, A. i. den Bewohnern der Insel Rhodus, den Apollo, den die

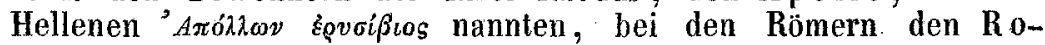

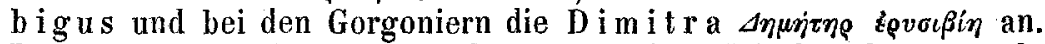
Der. Mehllhau, der den Früchten besonders Schaden thut, wurde bei den Alten 'Exáxviov genannt, indem sich derselbe in Form eines Thaues, $\pi \alpha x^{\prime} \chi \eta$ genannt, auf denselben zeigt.

Diese kryptogamischen Pflanzen waren bei den Alten gekannt. Ausserdem finden sich in Griechenland noch folgende: Pteris aquilina, - Asplenium Ruta muraria, Asp. flix foemina, Asp. Adiantum nigrum, - Marchantia polymorpha, - Funaria hygrometrica, Parmelia Roccella, P. articulata, - Lichen Prunaster, - Parmelia parietina, - Nostoc commune, - Tuber cibarium, - Agaricus muscarius, Ag. deliciosus, Ag. mutabilis, Ag. bombycinus, Ag. ignarius, Ag. Larias, - Tremella Auricula. - Alle diese Kryptogamen bleiben in Griechenland unbenützt.

\section{Ueber Vegetationsbeobachtungen.}

Im vorigen Winter wurden viele botanische Excursionen gemacht; heuer fallen die Schneeglöckchen vom Himmel. - Viele Verzeichnisse im Winter blühender Pflanzen wurden geschrieben; keines lat entsprochen! Ich selbst habe an keiner ausdauernden Pflanze, welche in Winter ihre Vegetation fortgesetzt oder präcipitirt hatte, in ihrem gewöhnlichen Gange irn nächsten Frühjahre einen Unterschied bemerkit. Die Rosskastanien, welche im Spätherbste 